\title{
ABUNDANCES IN RAPIDLY ROTATING A STARS
}

\section{MICHAEL LEMKE}

Astronomy Department, University of Texas, Austin, TX 78712; U.S.A.

Abstract. A study is undertaken to determine abundances in rapidly rotating main sequence A stars ( $v \sin i=100-180 \mathrm{~km} / \mathrm{s}$ ) in order to investigate if non-solar abundance patterns found in slow rotators (Lemke 1989, 1990) persist toward higher rotational velocities. This will provide some insight into how the Am phenomenon and other chemical peculiarities are connected with slow rotation and diffusion processes.

Most stars were found to have a rather solar composition. At least one star, $\mathrm{HR} 5023$, is enriched in $\mathrm{Ba}$ by approximately one order of magnitude relative to the sun at a $v \sin i$ of $110 \mathrm{~km} / \mathrm{s}$. Both $\mathrm{C}$ and $\mathrm{Ba}$ will be discussed.

\section{Observations}

A sample of some 20 rapidly rotating, supposedly normal A stars were observed with the $2.1 \mathrm{~m}$ and $2.7 \mathrm{~m}$ telescopes of McDonald Observatory, University of Texas, using the Coudé focus with conventional gratings and CCD detectors. The objects were selected from the Bright Star Catalogue (Hoffleit and Jascheck, 1982) according to the following criteria: spectral type AOV - A2 V; single stars or well separated binaries; $50 \mathrm{~km} / \mathrm{s}<v \sin i<150 \mathrm{~km} / \mathrm{s}$ (with two slower rotators included).

The stars were observed in up to 6 spectral bands, each approximately $60 \AA$ wide, with central wavelengths between $4224 \AA$ and $5050 \AA$. In addition, spectra were taken of the Hydrogen Balmer line $\mathrm{H}_{\beta}$ in low resolution, giving $400 \AA$ wide pieces of data. The program stars are listed in Table 1 together with spectral type and adopted parameters.

\section{Temperatures and Gravities: Evidence for Convective Core Overshoot?}

Effective temperatures were derived from Strömgren photometry while gravities were determined from both photometry and $\mathrm{H}_{\beta}$ line profiles. Photometric data were taken from Hauck and Mermilliod (1985) and converted by using the calibration of Moon and Dworetsky (1985). Gravities from Hydrogen line profiles were derived by interpolating the observed profile between two synthetic spectra, computed from ATLAS6 models with different gravities and the photometrically determined temperature.

The fitting procedure has an internal accuracy of \pm 0.01 to \pm 0.02 dex which makes it potentially much more precise than the photometric method. The fitting is not unique however-it also depends slightly on the adopted temperature, which then is the main source of uncertainty of the final gravity. 
Compared to the photometric data, the $\mathrm{H}_{\beta}$ fits generally give lower gravities, which also show a smaller spread yet extend to equal extreme values (compare left and right panel in Fig. 1).

Comparison of the stars' positions in a $\log g-\log T_{\text {eff }}$ diagram with evolutionary tracks computed with and without convective overshoot (Hejlesen, 1980, and Bertelli et al., 1986, respectively) give strong indication for overshoot parameters greater than 0 (Fig. 1). Similar results were found for other A-type stars by Andersen et al. (1990) and by Lemke (1989), as well as by Napiwotzki et al. (1991) for 'blue straggler' stars in NGC 2301.

The situation becomes less clear, however, when recent calculations by Castellani et al. (1990) are considered, which were made without core overshoot but taking new opacities into account. Their TAMS is indicated in Fig. 1, left panel. Quite a few of the program stars are still above that limit implying either only a small amount of overshoot is required or the adopted temperature scale is in error (as the gravity determination is sensitive to $T_{\text {eff }}$; see above). The latter cannot be ruled out from the current investigation: A comparison of the temperatures from the Moon\&Dworetsky calibration with those derived from least square fits to $T_{\text {eff }}$ vs. $c_{0}$ or vs. $a_{0}$ of data of Code et al. (1976) show systematically lower temperatures of approximately $150 \mathrm{~K}$ for the $\mathrm{M} \& \mathrm{D}$ grid. This would translate into higher gravities by $\sim 0.06 \mathrm{dex}$, which is marginally sufficient to move all stars (except HR 4194) onto Castellani's main sequence.

\section{Continuum determination}

Continuum fitting was done with a semi-automatic procedure of the FIGARO data reduction package, which fits polynomials to the observations and, based on these fits, successively rejects absorption lines until the continuum is found within the noise level. This procedure worked surprisingly well and the uncertainty of the continuum placement is probably only a secondary contributor to the total uncertainty of the abundances.

Yet, as in a rapidly rotating star all the lines get fairly much washed out, we have to deal with only a few percent variation of the continuum intensity. Due to the noise as well as to potentially non-unique line fits, the abundances can in most cases probably be estimated only within the 0.5 dex level. This should be sufficient, however, to detect the typical variations of $\mathrm{Ba}$ seen in sharp lined A stars.

\section{Synthetic Spectra and Rotational Velocities}

Severe line blending, caused by the rapid rotation of the stars, prohibits measurements of equivalent widths. Therefore, synthetic spectra were computed from ATLAS6 models using the gravities from the $\mathrm{H}_{\beta}$ fits. In a multivariate function fitting algorithm several abundances and the rotational velocitiy very varied simultaneously to find those values which yield the smallest $\chi^{2}$ of observed and 
synthetic spectrum indicating the optimal fit in an objective way. This method gives at the same time $v \sin i$ and abundances for a certain piece of spectrum.

Rotational velocities from different wavelength regions of the same star typically agree within less than $10 \%$, independent of what abundances were found. The resulting $v \sin i$ 's are fairly similar to those listed in the Bright Star Catalog, with only two major discrepancies: HR 3711 and HR 4861 both rotate about $100 \mathrm{~km} / \mathrm{s}$ faster.

\section{Abundances}

In this poster paper results are shown for the feature around the BaII $\lambda 4934 \AA$ resonance line. This line is blended by a neighboring $C_{1}$ line at $4932 \AA$ and a few negligible $\mathrm{Fe}$ lines (but included in the synthesis). Therefore, $\mathrm{Fe}, \mathrm{Ba}, \mathrm{C}$, and $v \sin i$ were allowed to float in the fitting procedure yielding abundances for these elements. Fe abundances are not meaningful at this point as there are a couple of very strong Fe lines which are very sensitive to the adopted microturbulence. The $\mathrm{Ba}$ and $\mathrm{C}$ lines, however, are rather weak and simulations for $\xi_{\text {micro }}=1$ and $\xi_{\text {micro }}=3 \mathrm{~km} / \mathrm{s}$ give abundances that differ by typically 0.1 dex only. Table 1 lists the results for $\mathrm{Ba}$ and $\mathrm{C}$ obtained with an adjusted microturbulence (between 0 and $3 \mathrm{~km} / \mathrm{s}$ ) that best fits the observed spectra.

Most stars were found to have an almost solar composition while a few of them might be enriched with $\mathrm{Ba}$ in a similar fashion as the slow rotators. Among the stars with a fairly high $v \sin i$, HR 5023 certainly has a Ba abundance which is about one order of magnitude higher than that of the sun. Its spectrum is shown in the upper half of Fig. 2 together with synthetic spectra for varied $\mathrm{Ba}$ abundances. For comparison, the lower half of the same figure displays the same region in HR 6610, a typical example for the more solar like stars. Variations of $T_{\text {eff }}$ by $400 \mathrm{~K}$ and of $\log g$ by 0.4 dex produce significantly less different spectra than does the increase of $\mathrm{Ba}$ by 0.5 dex. The effect of the blending $\mathrm{C}$ line can well be disentangled and is also automatically provided for by the fitting method.

Nevertheless, the $\mathrm{C}$ content is more uncertain-in particular as the slow rotators have mostly underabundances, which would make the line undetectably weak in the rapid rotators. The estimates given here indicate a variation independent of $\mathrm{Ba}$, which again is similar to the sharp lined stars.

Results for $\mathrm{Ba}$ and $\mathrm{C}$ as function of $v \sin i$ are summarized in Fig. 3.

Preliminary results for Fe reveal no striking variation except for HR 4861, which is certainly depleted between 1 and 1.5 dex relative to the sun. HR 3711 might also tend towards the same direction, putting both stars into the neighborhood of Vega. 


\section{References}

Andersen, J., Nordström, B., Clausen, J.V. 1990: Astrophys. J. 363, L33.

Bertelli, G., Bressan, A., Chiosi, C., Angerer, K. 1986: Astron. Astrophys. Suppl. 66, 191.

Castellani, V., Chieff, A., Straniero, O. 1990: Astrophys. J. Suppl. 74, 463.

Code, A.D., Davis, J., Bless, R.C., Hanbury Brown, R. 1976: Astrophys. J. 203, 417.

Hauck, B., Mermilliod, M. 1985: Astron. Astrophys. Suppl. 40, 1.

Hejlesen, P.M. 1980: Astron. Astrophys. Suppl. 39, 347.

Lemke, M. 1989: Astron. Astrophys. 125, 225.

Lemke, M. 1990: Astron. Astrophys. 240, 331.

Moon, T.T., Dworetsky, M.M. 1985: Monthly Notices Roy. Astron. Soc. 217, 305.

Napiwotzki, R., Schönberner, D., Weidemann, V. 1991: Astron. Astrophys. 243, L5.

Table 1: Program stars together with adopted parameters and $\mathrm{Ba}$ and $\mathrm{C}$ abundances.

\begin{tabular}{rlllrrrrc}
\hline HR & Name & Sp. Type & V & \multicolumn{1}{c}{$T_{\text {eff }}$} & $\log g$ & $v \sin i$ & Ba & C \\
\hline 830 & & A0 V & 5.86 & 10480 & 3.69 & 60 & 8.8 & 2.8 \\
945 & & A0 V & 6.42 & 9850 & 3.73 & 75 & 8.7 & 2.3 \\
1251 & $3813 \mathrm{Tau}$ & A1 V & 3.91 & 9230 & 3.87 & 80 & 8.3 & 2.5 \\
1661 & & A0 V & 6.05 & 10520 & 3.82 & 30 & 8.6 & 3.5 \\
3711 & & A1 V & 6.53 & 9770 & 3.74 & 230 & 7.3 & $1.9:$ \\
4131 & & A1 V & 6.45 & 9760 & 3.70 & 180 & 6.8 & 1.9 \\
4194 & & A0 V & 5.64 & 9730 & 3.55 & 90 & 8.5 & 1.8 \\
4635 & 3 CrV & A2 V & 5.46 & 9100 & 3.96 & 130 & 8.4 & 2.0 \\
4861 & 28 Com & A1 V & 6.56 & 9340 & 3.92 & 180 & 8.8 & - \\
5023 & 21 CVn & A0 V & 5.15 & 10750 & 3.93 & 110 & 8.7 & 3.5 \\
5262 & & A2 V & 5.99 & 8670 & 3.78 & 125 & 8.5 & 2.4 \\
6195 & $37 \mathrm{Her}$ & A1 V & 5.77 & 10110 & 4.00 & 160 & 8.7 & 2.9 \\
6610 & & A0 V & 6.56 & 9890 & 3.96 & 135 & 8.6 & 2.6 \\
6976 & & A1 V & 6.40 & 9900 & 3.71 & 180 & 8.5 & 2.8 \\
7351 & & A1 V & 6.26 & 9520 & 3.77 & 125 & 8.6 & 2.7 \\
8083 & & A0 V & 6.17 & 10610 & $4.25 *$ & 135 & 8.5 & $1.0:$ \\
8186 & & A1 V & 6.63 & 9510 & $4.02^{*}$ & 160 & 8.3 & $1.8:$ \\
\hline \hline
\end{tabular}

* from Strömgren colors

: uncertain 

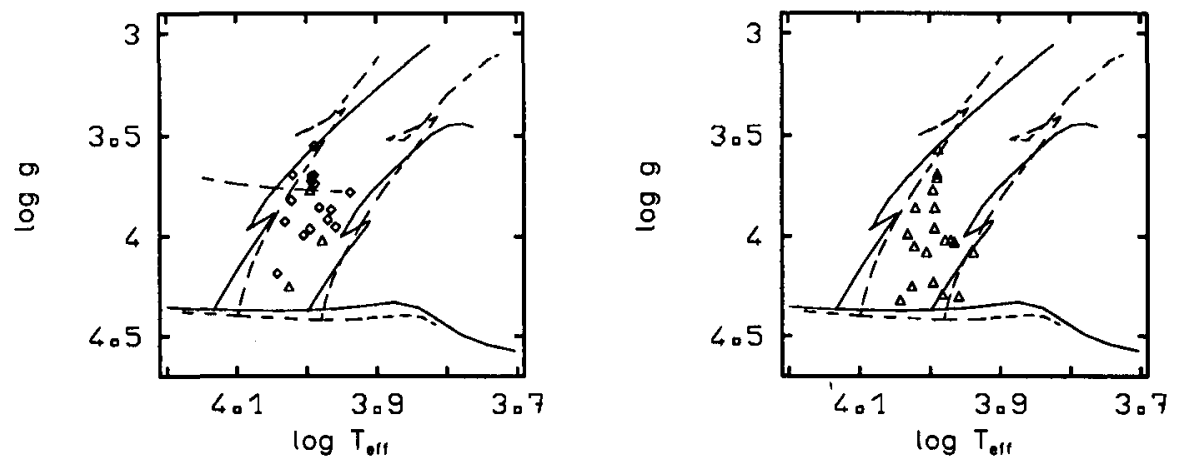

Figure 1: $\log g-\log T_{\text {eff }}$ diagrams with evolutionary tracks for 2 and 3 solar masses. $\diamond: \log g$ from $\mathrm{H}_{\beta}$ profiles; $\Delta$ : from photometry. Solid lines: Evolutionary data from Hejlesen (1980), broken lines: Bertelli et al. (1986); upper broken line (left panel): TAMS for solar composition from Castellani et al. (1990).

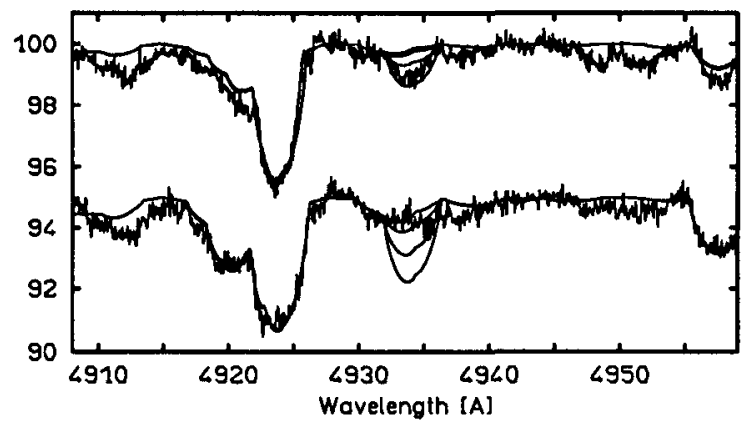

Figure 2: Spectrum for HR 5023 (up) and HR 6610 (shifted by 5\%) around the Ba II $\lambda 4934 \AA$ line plus synthetic spectra. Ba abundance increased in steps of 0.5 dex, starting from 2.18 (solar).
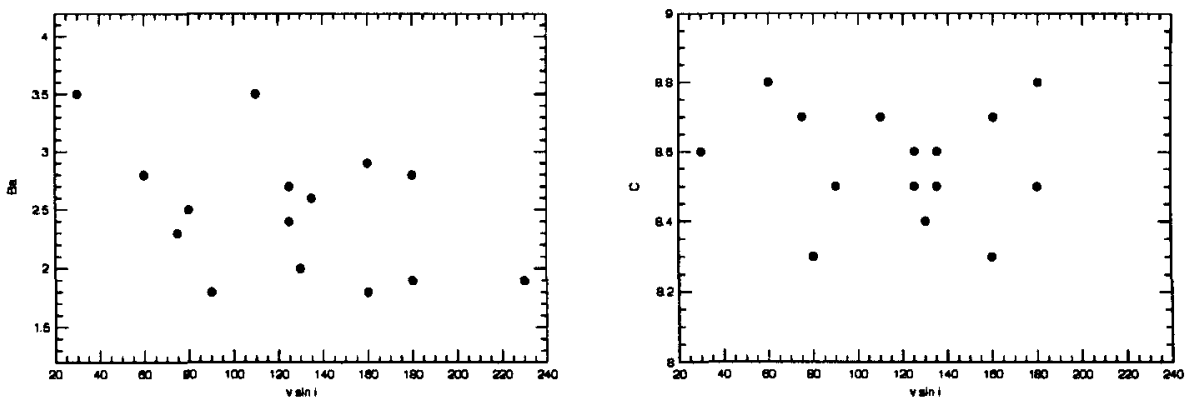

Figure 3: $\mathrm{Ba}$ (left panel) and $\mathrm{C}$ abundances as function of $v \sin i$. 


\section{DISCUSSION (Lemke)}

DWORETSKY: The greater spread of $\log g$ for early A stars, based on $u v b y \beta$ calibrations vs. detailed Balmer line analysis, is not surprising. The typical scatter for $\log g$ from $u v b y \beta$ is \pm 0.15 dex for individual stars. If you can use Balmer line profile fits to help determine $\log g$ you should regard this as having a higher weight than photometry alone.

LEMKE: Thank you for that comment.

SHORE: Could the Ba-enhanced stars that you found perhaps be main sequence Ba stars where the accretor is an A star? There should, according to Eggleton (1992, IAU Symp. 151) be some. He has performed simulations of the Bright Star Catalogue using binary evolution models and has argued that there must be quite a number of main sequence $\mathrm{Ba}$ stars.

NORTH: It would be interesting to look for an overabundance of $\mathrm{Sr}, \mathrm{Y}$ and $\mathrm{Zr}$, which are also enhanced in classical Ba stars. Do you have any results on such elements?

LEMKE: I am still working on the analyses, which include Sr, and if memory serves this star is enhanced in Sr. But the Sr lines are much stronger than the $\mathrm{Ba}$ feature and are sensitive to microturbulence, which is not yet known. Thus, these results are very preliminary. 